\title{
Novel Nanoparticle Biomaterial of Alginate/Chitosan Loading Simultaneously Lovastatin and Ginsenoside RB 1: Characteristics, Morphology, and Drug Release Study
}

\author{
Quan Vo-An, ${ }^{1}$ Thuy Chinh Nguyen $\mathbb{D}^{1,2}$ Quang Tung Nguyen ${ }^{1}{ }^{3}{ }^{3}$ Quoc Trung Vu, ${ }^{4}$ \\ Cong Doanh Truong, ${ }^{3}$ Thi Len Nguyen, ${ }^{3}$ Thi Ngoc Lien Ly, ${ }^{3}$ Long Giang Bach, \\ and Hoang Thai $\mathbb{D}^{1,2}$ \\ ${ }^{1}$ Institute for Tropical Technology, Vietnam Academy of Science and Technology, 18 Hoang Quoc Viet, Cau Giay, \\ Ha Noi 100000, Vietnam \\ ${ }^{2}$ Graduate University of Science and Technology, Vietnam Academy of Science and Technology, 18 Hoang Quoc Viet, Cau Giay, \\ Ha Noi 100000, Vietnam \\ ${ }^{3}$ Hanoi University of Industry, No. 298, Cau Dien Street, Bac Tu Liem District, Ha Noi 100000, Vietnam \\ ${ }^{4}$ Faculty of Chemistry, Hanoi National University of Education, No. 136 Xuan Thuy Road, Cau Giay, Ha Noi 100000, Vietnam \\ ${ }^{5}$ NTT Institute of High Technology, Nguyen Tat Thanh University, 300A Nguyen Tat Thanh, District 4, \\ Ho Chi Minh City 700000, Vietnam
}

Correspondence should be addressed to Quang Tung Nguyen; quangtungdhcnhn@gmail.com and Hoang Thai; hoangth@itt.vast.vn

Received 8 June 2020; Revised 4 August 2020; Accepted 21 August 2020; Published 16 January 2021

Guest Editor: Yuting Huang

Copyright (c) 2021 Quan Vo-An et al. This is an open access article distributed under the Creative Commons Attribution License, which permits unrestricted use, distribution, and reproduction in any medium, provided the original work is properly cited.

\begin{abstract}
Recently, plenty of interesting studies on improvement of bioavailability for poorly soluble drugs were implemented with different approaches such as using of combined biopolymers as a delivery system that allowed to enhancing drug solubility and bioavailability. In this work, alginate and chitosan were blended together in the form of polymeric particles, loaded with both lovastatin and ginsenoside $\mathrm{Rb} 1$ to producing the four-component nanoparticles by ionic gelation method. $\mathrm{CaCl}_{2}$ and sodium tripolyphosphate were used as gelation agent and cross-linking agent, respectively. The characteristics of obtained nanoparticles were studied by means of infrared spectra (IR), scanning electron microscopy (SEM), differential scanning calorimetry (DSC), and dynamic light scattering (DLS). In combination, ginsenoside Rb1 and lovastatin both interacted with each other to improve the drug release ability of the polymer particles. The change of initial content of drugs in the nanoparticles has a negligible effect on the functional groups in the structure of the nanoparticles but has a significant impact on drug release process of both lovastatin and ginsenoside $\mathrm{Rb} 1$ from the nanoparticles in selective simulated body fluids. In addition, the synergistic interaction of lovastatin and ginsenoside Rb1 could be also observed through the modification of relative crystal degree and drug release efficiency.
\end{abstract}

\section{Introduction}

Alginate (AG) and chitosan (CS) are two common natural polymers with very diverse applications in different fields such as medical, pharmaceutical, food, beverage, and arts. AG is found in 265 generals of brown algae's [1] as an organic acid with molecular weight of 32,000-200000 which was discovered by Stanford in the late 19 th century $[1,2]$. AG dissolves into water and forms a highly viscosity aqueous solution that is helpful for food preservation to enlarge the storage time while keeping the quality [3-8]. Moreover, AG has also been used in paper industry [9], biomedical 
applications [3, 10, 11], wound management [12-14], and fiber industry [15] for its convenient-to-use, nontoxic characteristic.

Chitosan (CS), scientific name poly(1,4)-2-amino-2deoxy- $\beta$-D-glucose or poly $(1,4)$-2-amino-2-deoxy- $\beta$-D-glucopyranose, is a deacetylate derivative of chitin-the second abundant polysaccharide in nature (after cellulose). It is also the second most popular biopolymer in the world [16] with large application in food industry $[17,18]$, agriculture [19-21], cosmetic [18], water treatment technology $[17,18,22]$, and biomedical materials and pharmaceuticals [23-25]. Especially, CS is also a pivotal material in preparation glucosamine $[26,27]$.

The combination of these two abovementioned materials gives remarkable enhancement in antibacterial properties both for AG bandages as well as AG fibers while coming with CS [15]. Thus, the studies on fabricating and applying AG/CS polymer blend as material for loading drugs are now an attractive research direction.

Lovastatin (Lov) is a naturally occurring fermentation compound that was discovered in 1970. It was found in red yeast rice [28] and oyster mushrooms [29]. Lov inhibits cholesterol biosynthesis, reduces cholesterol in liver cells, and stimulates synthesis of LDL (low-density lipoprotein) receptors, thereby increasing LDL transport from blood to reduce plasma cholesterol levels. Thus, the prevention of cardiovascular diseases and treatment of dyslipidemia are main applications of Lov [30]. However, Lov has an average decomposition half-time approximately 3-4 hours, so patients have to take it several times a day to maintain the minimum drug level. To overcome this limitation, Lov is loaded into polymers that have the ability to control drug release through physical interactions between polymers and drug, decreasing number of drug using times. In the work on potential nanochitosan materials carrying Lov at different concentrations of CS, Shinde has figured out that Lov is absorbed by CS and the ability of drug loading decreases while increasing CS concentration. CS materials loading with Lov had shown its effectiveness in maintaining drug release for a long time [31]. Other studies on different drug-carrier polymer systems have also shown the same advantage in controlling drug release of these polymers [32-37]. Our previous studies have been done by preparing and releasing Lov from AG/CS polymers in both film materials [38] and nanoparticles [39], in which, the size of AG/CS/Lov nanoparticles was 50-100 nm. The efficacy of Lov release control was evident in the later stages, and the Lov release process from AG/CS/Lov nanoparticles had the most suitable Korsmeyer-Peppas kinetic model with complex mechanism [39].

Panax notoginseng is a well-known folk medicinal herbal using for the treatment of hemostatic, hemoptysis, and hematoma for centuries in China, Vietnam, and other Asian countries [40-44]. Over 200 chemical compounds have been isolated from the plant, but the main constituents are Panax notoginseng saponins (PNS) $[39,45]$. Two major saponins as ginsenoside Rb1 and ginsenoside Rg1 have been identified from the PNS harvested in Vietnamese (Vietnamese Panax pseudoginseng). While ginsenoside Rg1 has stimulating effect on nerves system, antifatigue, improves brain work, ginseno- side $\mathrm{Rb} 1$ has the effect of inhibiting nerves center for sedation and deep sleep. In our previous study, a composite film combining AG/CS material and ginsenoside Rb1 had been made by solution method, and results that AG, CS, and ginsenoside Rb1 had strongly interacted to each other and created a polyelectrolyte complex [46].

In the recent work, the drug delivery polymer system of AG/CS polymer blend with 5 wt.\% ginsenoside Rb1 and different Lov contents in film form was produced and studied. The results showed that Lov and ginsenoside Rbl had a synergistic effect which affect on the morphology, properties, and drug release ability of these polymer films [45]. In preparation of AG/CS nanocomposites loading with Lov and ginsenoside Rb1 by ionic gelation method, the impact of both calcium chloride as a gel forming substance and sodium tripolyphosphate (STPP) as a cross-linking agent to the properties of blend polymer was also reported [47]; from those, the most suitable concentration of calcium chloride and STPP was found.

Unlike the 3-components of AG/CS nanocomposites loading only with either Lov or ginsenoside Rb1, the synergism between Lov and ginsenoside Rb1 and AG/CS clearly appeared in the 4-component composite while in film form. This synergistic effect improved both of the drug loading ability and the releasing efficiency, overcoming the disadvantages of the 3-component nanocomposite such as limitation of drug release only reach $80-85 \%$ [39]. That caused the large amount of used drugs to be wasted.

Combining between the excellent features of each component as well as the results of previous studies, the simultaneous load of ginsenoside Rb1 and Lov into the drug delivery polymer system of AG/CS has proved to give a better result to the drug release process from AG/CS/Lov/ginsenoside Rb1 composite in film form $[45,46]$.

In order to inherit and promote the before-mentioned advantages and aiming to enhance practical application, a 4-component composite system with small size has been fabricated to facilitating surface interaction between the components, leading improvement of drug loading capacity and drug release efficiency.

In this paper, novel nanoparticle biomaterial of AG/CS loading simultaneously Lov and ginsenoside Rb1 (NPB) is produced by ionic gelation method in favorable condition. The physical characteristics, morphology, drug loading efficiency, and drug release process of obtained NPB have been studied and discussed.

\section{Experimental}

2.1. Materials. Chitosan (CS) powder with deacetylation degree of $75 \%$, sodium alginate powder (AG) with viscosity 300-500 mPa.s, lovastatin powder (Lov) with purity of 98.0\%, and sodium tripolyphosphate (STPP) were commercial chemicals from Sigma-Aldrich. Ginsenoside Rb1 in white powder with the purity of $98 \%$ was extracted from Panax pseudoginseng and provided by National Institute of Medicinal Materials, Vietnam. Other analytical grade chemicals and solvents are ethanol, acetic acid 1\%, and calcium chloride $\left(\mathrm{CaCl}_{2}\right)$. 


\subsection{Preparation of $A G / C S / L O V / G i n s e n o s i d e ~ R b 1$ Nanoparticle} Biomaterials (NPB) by Ionic Gelation Method. An ionic gelation method with $\mathrm{CaCl}_{2}$ gelation agent and STPP crosslinking agent has been used for the procedure of nanoscale to microscale polymer granulars. This technique is favorable because of simple preparation in aqueous solution. The gelation process was conducted basing on the polymer characteristic to establishing cross-linking bonds in the presence of ions, resulting in the formation of nanoparticles. In this method, CS has been dissolved in acetic acid solution to obtain CS cation. Afterward, the cation solution has been added slowly under continuous stirring into an AG solution containing STPP. Due to the interaction between the opposite charges, AG/CS undergoes cross-linking and gel-ionization, then precipitates to forming spherical particles. This NPB was remarkably effective to enhance health and immunity and may reduce blood cholesterol concentration and treat cardiovascular disease in patients.

2.3. Preparation of NPB with Varied Lov and Ginsenoside Rb1 Content. The preparation process is similar to the production of 4-component AG/CS/Lov/ginsenoside Rb1 with a selected concentration of $\mathrm{CaCl}_{2}$ of $0.002 \mathrm{M}$. The Lov content ranges from $0 \mathrm{wt} . \%, 10 \mathrm{wt} . \%, 15 \mathrm{wt} . \%$, to $20 \mathrm{wt} . \%$ (in comparison to the total mass of $\mathrm{AG}$ and $\mathrm{CS}$ ); $\mathrm{AG} / \mathrm{CS} /$ ginsenoside $\mathrm{Rb} 1$ ratio is $1: 1: 5 \mathrm{wt} . \%$, ginsenoside $\mathrm{Rb} 1$ content ranging from 0 to $5 \mathrm{wt} . \%$. Table 1 presents the composition and label of the different NPB samples.

The calculated CS, AG, Lov, and ginsenoside Rb1 were dissolved in acetic acid 1\% solution, distilled water, and ethanol 99\%, respectively. An STPP solution of $0.83 \mathrm{~g} / \mathrm{L}$ and $\mathrm{CaCl}_{2}$ aqueous $0.002 \mathrm{M}$ solution were also prepared.

After obtaining the above solution, mixing the STTP and $\mathrm{CaCl}_{2}$ solutions and stirring in high speed ultrasonic equipment at 20,000 rpm to increase the dispersion of the substances.

Gradually pour Lov solution to ginsenoside $\mathrm{Rb} 1$ and stir with high speed ultrasonic until reaching homogeneous mixture. Next, slowly drop the obtained STTP and $\mathrm{CaCl}_{2}$ solutions into the Lov and ginsenoside $\mathrm{Rb} 1$ mixture and keep stirring to produce the mixture of drug.

The loading process of drug into the AG/CS mixture was performed by slowly add the mixture of drug to the mixture of AG and CS solutions, with dripping rate at $3 \mathrm{~mL} / \mathrm{min}$ and keep stirring on high speed ultrasonic stirrer for $30 \mathrm{~min}$.

The final product was then collected after going through the two stages of solvent removal, centrifugation, and freezedry. The FreeZone 2.5 (Labconco, USA) was used as a freezedried equipment to evaporate completely the remaining solvent out of the materials. It was then minced into fine powder and stored in a cool, dry place before use.

\subsection{Standard Curve of Lov and Ginsenoside Rb1 in Different pH Solutions and in Ethanol}

2.4.1. Standard Curve of Lovastatin and Ginsenoside Rb1 in Buffer and in Ethanol. The standard curve and regression coefficients of Lov and ginsenoside $\mathrm{Rb} 1$ in different $\mathrm{pH}$ solutions ( $\mathrm{pH} 2$ and $\mathrm{pH} 7.4$ ) and in ethanol are established based on the data from the UV-Vis spectra. The Lov or ginsenoside $\mathrm{Rb} 1$ was dissolved in buffer or ethanol solution and stirred and measured the UV spectra data of each solution with different concentrations. The standard curve equations and linear regression coefficients $\left(R^{2}\right)$ were then calculated, in which $x$ and $y$ according to the optical absorbance (A) and concentration (C) of Lov and ginsenoside Rb1 (Table 2).

The regression coefficients of the standard curve equations presented in Table 2 are about $\sim 1$, which should be used to determine the efficiency of the Lov and ginsenoside carrying as well as their content released from the NPB.

2.4.2. Determination of Lov and Ginsenoside Loading Efficiency. As opposed to the Lov and ginsenoside loading process, their release process has been carried out by putting a certain amount of Lov or ginsenoside Rb1 loaded nanoparticle into ethanol solution and stirring well for 24 hours. An exact volume of the solution was filtered and used to determining the optical density of Lov and ginsenoside Rbl. This data was used to comparing and calculating based on the standard curves of Lov and ginsenoside Rb1 in ethanol to obtain the loading efficiency of Lov and ginsenoside Rb1 in NPB samples.

2.4.3. Evaluation of Lov and Ginsenoside Rb1 Release Process from $N P B$. The experiments of Lov and ginsenoside $\mathrm{Rb} 1$ release from NPB were conducted in the buffer solution at $\mathrm{pH} 2$ and $\mathrm{pH}$ 7.4. A certain amount of the drug loading nanoparticle was taken into buffer at $37^{\circ} \mathrm{C}$ while continuously stirred at 20,000 rpm. After every hour, a certain volume of the solution was taken to filter and recorded UV data of Lov and ginsenoside $\mathrm{Rb} 1$ concentration. An equivalent amount of fresh buffer solution had been added to maintain the initial volume. Similar procedure was then repeated until finishing 32 testing hours.

The amount of Lov and ginsenoside $\mathrm{Rb} 1$ released from $\mathrm{NPB}$ was calculated in corresponding to the standard curve equation of Lov and ginsenoside Rb1 in buffer solution at $\mathrm{pH} 2$ and $\mathrm{pH}$ 7.4. With each of Lov and ginsenoside $\mathrm{Rb} 1$, the percentage of them released at a time $(t)$ is calculated by the following formula:

Percentage of released Lov or ginsenoside Rb1 $(\%)=\left(\frac{C_{(t)}}{C_{(0)}}\right) * 100$,

where $C_{(0)}$ is the initial amount of loaded Lov or ginsenoside $\mathrm{Rb} 1$ and $C_{(t)}$ is the amount of Lov or ginsenoside $\mathrm{Rb} 1$ released at a time $(t)$.

\subsection{Morphology and Characteristics of NPB}

2.5.1. FTIR Spectra. Fourier Transform Infrared (FTIR) spectra of AG, CS, Lov, ginsenoside Rb1, and drug-loading nanoparticles were recorded on a Nicolet iS10 spectrometer (Thermo Scientific, USA) at room temperature by 32 scans with $8 \mathrm{~cm}^{-1}$ resolution and wavenumber ranging from 400 to $4000 \mathrm{~cm}^{-1}$. 
TABLE 1: Composition and label of NPB samples.

\begin{tabular}{|c|c|c|c|c|}
\hline \multicolumn{5}{|c|}{$\begin{array}{l}\text { Samples label- }-\mathrm{R}_{\mathrm{x}} \mathrm{L}_{\mathrm{y}} \text { (ratio of initial content of } \\
\text { ginsenoside } \mathrm{Rb} 1 \text { and Lov in NPB) }\end{array}$} \\
\hline \multirow{4}{*}{\multicolumn{3}{|c|}{ Ginsenoside Rb1 initial content (wt.\%) }} & $0 \%$ & \\
\hline & & & $1 \%$ & \\
\hline & & & $3 \%$ & \\
\hline & & & $5 \%$ & $\mathrm{R}$ \\
\hline \multicolumn{5}{|c|}{$\begin{array}{l}\text { TABLE 2: LOV and ginsenoside Rb1 standard curve equations in } \\
\text { different } \mathrm{pH} \text { solutions and in ethanol. }\end{array}$} \\
\hline \multirow{2}{*}{ Drugs } & \multirow{2}{*}{ Medium } & \multicolumn{3}{|c|}{$\begin{array}{c}\text { Parameters of standard curve equation } \\
\text { of drugs }\end{array}$} \\
\hline & & $\begin{array}{l}\text { Standard curve } \\
\text { equation }\end{array}$ & $R^{2}$ & $\begin{array}{l}\lambda_{\max } \\
(\mathrm{nm})\end{array}$ \\
\hline \multirow{3}{*}{ Lov } & $\mathrm{pH} 2$ & $y=6187 x+0.011$ & 0.996 & 229.97 \\
\hline & pH 7.4 & $y=3197.2 x+0.019$ & 0.996 & 239.15 \\
\hline & Ethanol & $y=31269 x+0.3012$ & 0.993 & 245.55 \\
\hline \multirow{3}{*}{$\begin{array}{l}\text { Ginsenoside } \\
\text { Rb1 }\end{array}$} & $\mathrm{pH} 2$ & $y=5400 x+0.0196$ & 0.996 & 219.52 \\
\hline & $\mathrm{pH} 7.4$ & $y=2077 x+0.014$ & 0.996 & 219.00 \\
\hline & Ethanol & $y=4513.8 x+0.002$ & 0.991 & 213.55 \\
\hline
\end{tabular}

2.5.2. Scanning Electron Nanoscopy (SEM). SEM images of the NPB were taken on a FESEM S4800 device (Hitachi, Japan) with magnification from 1000 times to 200000 times. The samples were coated with $\mathrm{Pt}$ to improve the conductivity of materials.

2.6. Differential Scanning Calorimetry (DSC). Schematic DSC curves of the NPB were measured on DSC-60 (Shimadzu, Japan) with heating condition from room temperature to $400^{\circ} \mathrm{C}$, heat rate at $10^{\circ} \mathrm{C} / \mathrm{min}$ in gaseous nitrogen.

2.6.1. Dynamic Light Scattering (DLS). The Zetasizer SZ-100 equipment was used to determine particle size of NPB at $25^{\circ} \mathrm{C}$, with measuring range from 0.1 to $10000 \mathrm{~nm}$. The NPB was dispersed in distilled water before recording the size distribution diagram.

2.6.2. Ultraviolet-Visible Spectroscopy (UV-VIS). The UV-Vis spectrometer (Cintra 40, GBC, USA) was used to determine the loading efficiency and concentration of Lov, ginsenoside $\mathrm{Rb} 1$ released from the NPB in different $\mathrm{pH}$ solutions and in ethanol.

\section{Results and Discussion}

3.1. FTIR Spectra of NPB. The FTIR spectra of AG, CS, Lov, and ginsenoside $\mathrm{Rb} 1$ are presented in Figure 1. Characterized bands of functional groups in $\mathrm{AG}$ are listed as follows: stretching $\mathrm{C}-\mathrm{H}$ bonding at $2923.65 \mathrm{~cm}^{-1} ; \mathrm{C}=\mathrm{O}$ group at $1626.64 \mathrm{~cm}^{-1}$; C-O-C bending vibration at $1096.95 \mathrm{~cm}^{-1}$ and $1032.1 \mathrm{~cm}^{-1}$; and the saccharide ring vibrations at $947.86 \mathrm{~cm}^{-1}, 891.80 \mathrm{~cm}^{-1}$, and $824.34 \mathrm{~cm}^{-1}$. A broad band at $3448.16 \mathrm{~cm}^{-1}$ represents the stretching vibrations of $-\mathrm{OH}$ group in AG macromolecule.

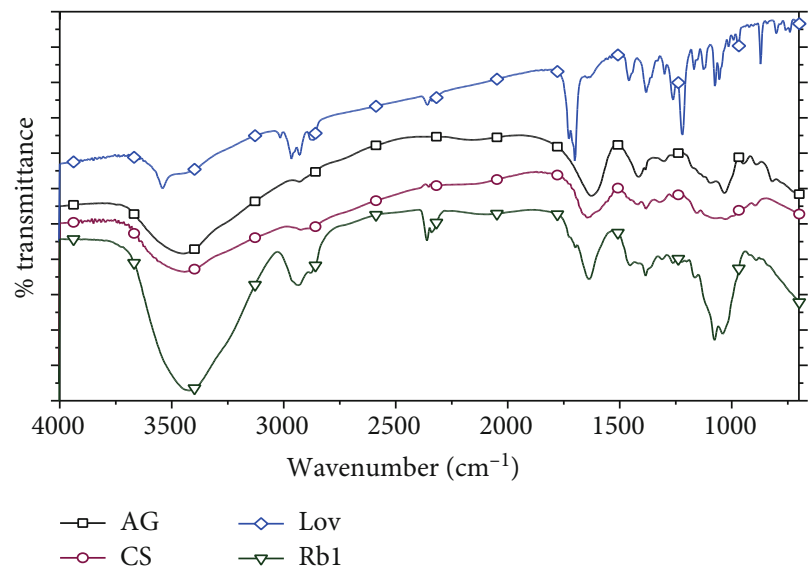

Figure 1: FTIR spectra of AG, CS, Lov, and ginsenoside Rb1.

In the FTIR spectrum of CS, the characteristic peaks of $\mathrm{CH}$ and $\mathrm{C}-\mathrm{O}$ groups are at $2883.51 \mathrm{~cm}^{-1}$ and $1082.75 \mathrm{~cm}^{-1}$; a broad peak at $3447.62 \mathrm{~cm}^{-1}$ attributed to $-\mathrm{OH}$ and $-\mathrm{NH}_{2}$ groups and amide group at $1598.29 \mathrm{~cm}^{-1}$ which is assigned for $-\mathrm{NH}_{2}$ group; other peaks also appear at $895.17 \mathrm{~cm}^{-1}$ for saccharide ring vibration in CS and at $1651.26 \mathrm{~cm}^{-1}$ for $\mathrm{C}=$ O stretching.

In the FTIR spectrum of ginsenoside $\mathrm{Rb} 1$, the broad band at $3423.35 \mathrm{~cm}^{-1}$ represents for $-\mathrm{OH}$ stretching vibration and $2937.96 \mathrm{~cm}^{-1}$ and $2941.92 \mathrm{~cm}^{-1}$ band for $\mathrm{CH}_{2}$ and $\mathrm{CH}$ stretching. The stretching vibration of $\mathrm{C}=\mathrm{C}$ bonding appeared in $1640.73 \mathrm{~cm}^{-1}$ band. The absorption band at $1077.79 \mathrm{~cm}^{-1}$ is for $\mathrm{C}-\mathrm{O}-\mathrm{C}$ stretching and the $-\mathrm{OH}$ bending vibration at $1364.06 \mathrm{~cm}^{-1}$ band.

The IR spectrum of pure Lov has characterized groups such as $-\mathrm{OH}$ stretching vibration at $3640.99 \mathrm{~cm}^{-1}$; the stretching vibration of $\mathrm{C}-\mathrm{H}$ bonding in $-\mathrm{CH}_{3},-\mathrm{CH}_{2^{-}}$, and $-\mathrm{CH}$ - appeared in three bands at $2966 \mathrm{~cm}^{-1}, 2929 \mathrm{~cm}^{-1}$, and $2867 \mathrm{~cm}^{-1}$; and $-\mathrm{C}=\mathrm{O}$ stretching vibration of saturated lactones at $1728.02 \mathrm{~cm}^{-1}$. The absorption band at $1696 \mathrm{~cm}^{-1}$ characterized for the stretching vibration of $\mathrm{C}=\mathrm{C}$ bonding of Lov, and the $1701.11 \mathrm{~cm}^{-1}$ band is due to $\mathrm{C}=\mathrm{O}$ group in the Lov structure. The C-O-C stretching is at $1074.50 \mathrm{~cm}^{-1}$.

The similarity has been found in the FTIR spectrum of other NPB-RxLy in comparison with the R5L10 sample (Figures 2 and 3). Thus, only the above characterized peaks of functional groups in the samples have been listed in Table 3.

The results of FTIR analysis of NPB-RxLy show that the peak position of the characterized groups in AG, CS, Lov, and ginsenoside $\mathrm{Rb} 1$ has shifted slightly due to the mutual 


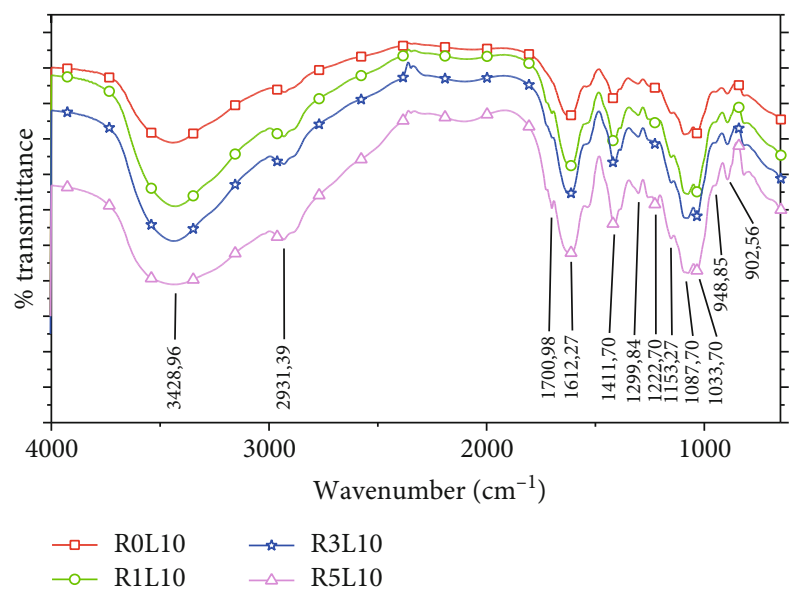

FIgURE 2: FTIR spectra of NPB-RxL10.

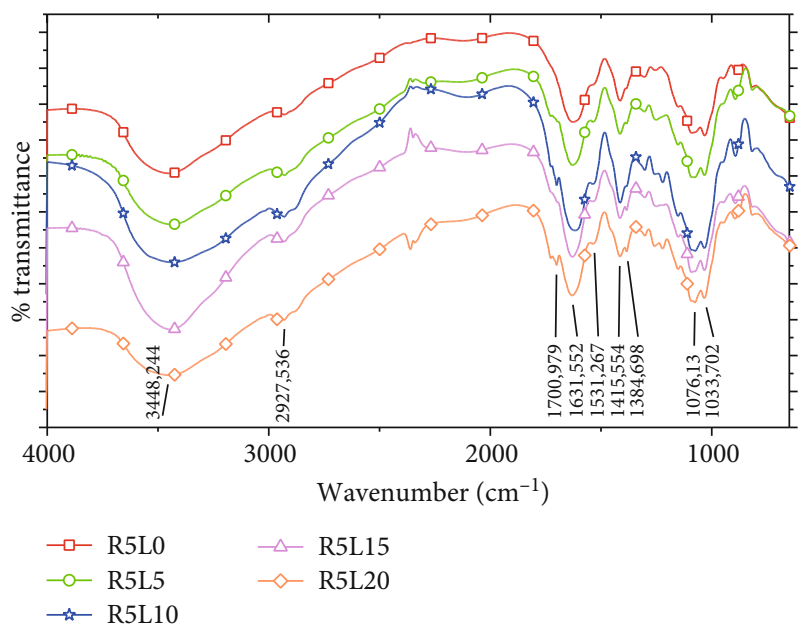

FIGURE 3: FTIR spectrum of NPB-R5Ly.

interaction between the characteristic groups in AG, CS, Lov, and ginsenoside $\mathrm{Rb} 1$ (polymer-polymer, polymer-drug, and drug-drug interactions). Following the FTIR spectra, the position of functional groups of 4 components in the NPB was a negligible change as adding or raising Lov's content while the intensity of these bands had varied. Thus, when using STPP as cross-linking agent, the band of the characterized groups in the NPB is significantly shifted; it might due to the better interaction and mixing of the components in NPB.

3.2. Morphology of NPB. FESEM images of Lov and ginsenoside $\mathrm{Rb} 1$ are displayed in Figure 4. Lov structure has a rodshaped, irregular size about $20 \mu \mathrm{m}-30 \mu \mathrm{m}$, and ginsenoside $\mathrm{Rb} 1$ has granular type with irregular size about $10 \mu \mathrm{m}-15 \mu \mathrm{m}$.

In Figure 5(f), in the NPB-R0L10 sample (without Rb1), the size of the collected particles is relatively large; phase separation appeared; the Lov rod dispersed unevenly with $1.5 \mu \mathrm{m}$ size and agglomerated into cluster, while the initial size of Lov rod is about $20-30 \mu \mathrm{m}$. Thus, just only loading in AG/CS, combined with a fabrication method with STPP as a cross-linking agent and high-speed ultrasonic stirring, the Lov rod sizes have been reshaped into much smaller.
TABLE 3: IR band for characterized groups of AG, CS, Lov, ginsenoside Rb1, and NPB with different Lov content.

\begin{tabular}{lccccc}
\hline \multirow{2}{*}{ Sample } & \multicolumn{5}{c}{ Wavenumber $\left(\mathrm{cm}^{-1}\right)$} \\
& $v_{\mathrm{NH} 2 . \mathrm{OH}}$ & $v_{\mathrm{CH}}$ & $v_{\mathrm{C}=\mathrm{O}}$ & $\delta_{\mathrm{NH} 2}$ & $v_{\mathrm{C}-\mathrm{O}-\mathrm{C}}$ \\
\hline Alginat (AG) & 3448.16 & 2923.65 & 1626.64 & - & 1096.95 \\
Chitosan (CS) & 3447.62 & 2883.51 & - & 1598.29 & 1081 \\
Lovastatin (Lov) & 3640.99 & 2966.06 & 1728.11 & - & 1077.79 \\
Ginsenoside Rb1 & 3423.35 & 2941.92 & 1077.79 & 1451.91 & 1070 \\
R5L0 & 3451.51 & 2928.31 & 1627.43 & 1414.17 & 1089.41 \\
R0L10 & 3443.16 & 2928.37 & 1621.10 & 1416.30 & 1090.57 \\
R3L10 & 3440.53 & 2931.39 & 1619.98 & 1415.55 & 1033.70 \\
R5L10 & 3426.96 & 2931.39 & 1616.12 & 1415.55 & 1091.56 \\
R5L20 & 3441.66 & 2968.62 & 1628.84 & 1535.36 & 1075.37 \\
\hline
\end{tabular}

With 5\% ginsenoside Rb1 (R5Ly), the rod/particle size of Lov in 4-component NPBs has been significantly reduced but still not evenly dispersed into the AG/CS polymer blend, resulting in the size of about $200 \mathrm{~nm}-1 \mu \mathrm{m}$ observed. From Figures 5(b)-5(e), as the Lov content increases, it seems that the impact of drug-drug interaction exceeds the polymerdrug interaction, and the drug tends to agglomerate to form larger size rods/particles.

Figures 6(a) and 6(b) show the SEM image of R0L10 surface at magnifications of 10,000 times and 30,000 times. The polymer particles appeared with relatively large size and heavy rough surface; the Lov rod was $1.5 \mu \mathrm{m}$ size and agglomerated together, much smaller in comparison with the size of pure Lov rod (Figure 4) ranging from $20 \mu \mathrm{m}$ to $30 \mu \mathrm{m}$.

Figures 6(c) and 6(d) present SEM image of NPB with 1 wt.\% ginsenoside Rb1 (R1L10 sample). The sizes of dispersed Lov rods are from 0.5 to $1 \mu \mathrm{m}$, and the particles are observed to be detached with each other. With the presence of ginsenoside $\mathrm{Rb} 1$, the Lov tends to have better dispersion in $\mathrm{AG} / \mathrm{CS}$ polymer particles.

In Figures 6(e) and 6(f), the SEM image of R3L10 using 3 wt.\% ginsenoside Rb1, the Lov rods have been covered with a polymer layer (brighter part surrounds the particles). Lov is better dispersed into AG/CS polymer blend in the size of 100$300 \mathrm{~nm}$. With $5 \mathrm{wt} . \% \mathrm{Rb} 1$ content (Figures $6(\mathrm{~g})$ and $6(\mathrm{~h})$ ), the Lov rods tend to agglomerate together and form larger particles, about $200 \mathrm{~nm}-1 \mu \mathrm{m}$.

It is very likely that the increase in ginsenoside $\mathrm{Rb} 1$ content also increases the internal molecular linkage between ginsenoside $\mathrm{Rb} 1$ molecules, thus, leading to the aggregation of ginsenoside $\mathrm{Rb} 1$ particles, reducing their size stability of ginsenoside $\mathrm{Rb} 1$, and generating uneven structure in the NPB. Therefore, in addition to its biological activities, ginsenoside Rb1 might act as size stabilizer in NPBs through dipole-dipole interactions and hydrogen bonding $[45,46]$.

3.3. Thermal Characteristics of AG, CS, Lov, Ginsenoside Rb1, and NPB. DSC diagrams of NPB with 10\% Lov (R0L10) and $5 \%$ ginsenoside $\mathrm{Rb} 1$ (R5L0) are presented in Figure 7. Figure 8 (a) presents DSC diagrams of R5Ly group with a fixed content of 5 wt.\% ginsenoside Rb1. And Figure 8(b) 


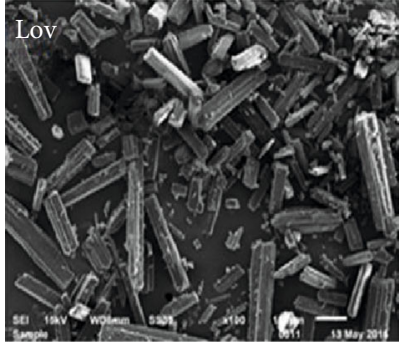

(a)

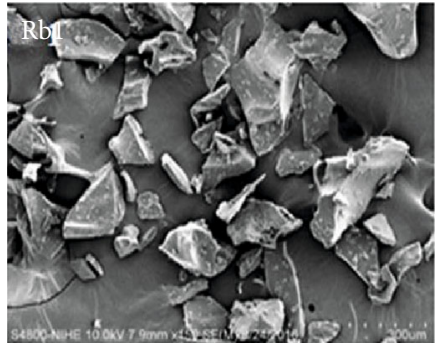

(b)

FIGURE 4: FESEM image of Lov (a) and ginsenoside Rb1 (b).

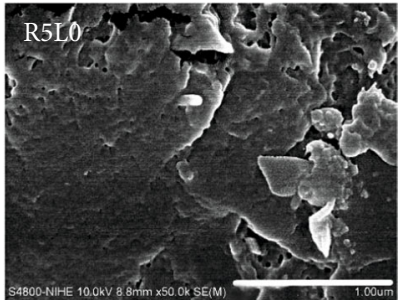

(a)

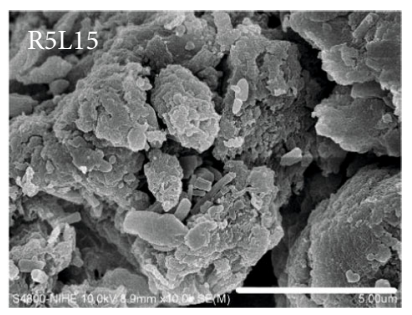

(d)

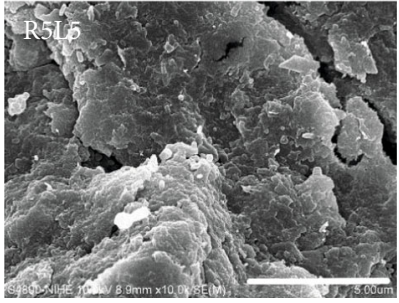

(b)

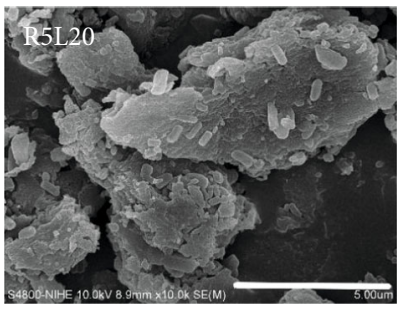

(e)

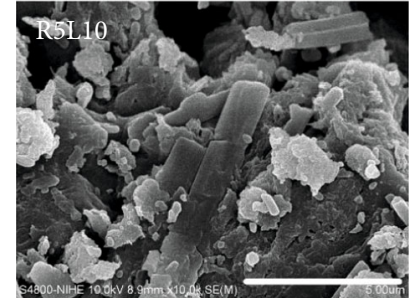

(c)

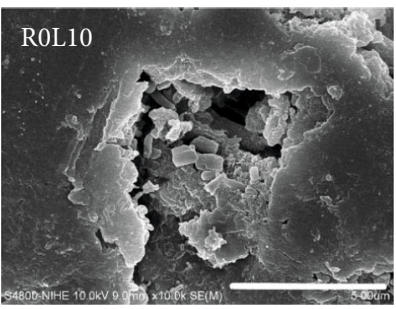

(f)

Figure 5: FESEM image of NPB with ginsenoside Rb1 5 wt.\% and (a-e) Lov 0, 5, 10, 15, and 20 wt.\% and (f) Lov 10 wt.\%.

presents DSC diagrams of RxL10 NPB group with 10\% Lov. The endothermic peak temperatures and exothermic peak temperatures of AG, CS, Lov, ginsenoside Rb1, and NPBs are listed in Table 4.

In Figure 7, the R5L0 sample has its two endothermic peaks at $89.51^{\circ} \mathrm{C}$ and $214.63^{\circ} \mathrm{C}$ corresponding to the dehydration and melting of AG and CS in NPB. For the R0L10 sample, two endothermic peaks located at $73.24^{\circ} \mathrm{C}$ and $221.74^{\circ} \mathrm{C}$ were also attributed to the dehydration and melting temperature of AG and CS. Moreover, one exothermic peak at $172^{\circ} \mathrm{C}$ can be assigned to the decomposition of STPP in the sample. Due to the forming a eutectic system between ginsenoside $\mathrm{Rb} 1$ or Lov, CS, and AG, the melting temperature of the NPB was decreased significantly as compared to the single component.

In the DSC diagrams of the R5Ly group (Figures 8(a) and 8(b)), the two endothermic peaks appeared because of the dehydration process at the temperature under $100^{\circ} \mathrm{C}$ and melting process of components in the samples at the temperature around $210^{\circ} \mathrm{C}$. Other two exothermic peaks are assigned for the decomposition of STPP cross-linking agent at the temperature about $174^{\circ} \mathrm{C}$ and the decomposition of $\mathrm{AG}$, Lov, and ginsenoside Rb1 at the temperature above $300^{\circ} \mathrm{C}$. For the R5L5 sample, the positions of two endother- mic peaks at $101.14^{\circ} \mathrm{C}$ and $218.65^{\circ} \mathrm{C}$ and one exothermic peak at $174.81^{\circ} \mathrm{C}$ are slightly shifted in comparison with provided DSC peaks of AG, CS, Lov, and the 3-components R5L0 and R0L10 (Table 4). This result showed that the changes of initial Lov content or ginsenoside Rb1 content have a significant effect on phase transition of the NPB samples. In this case, due to synergistic effect, all the recorded DSC peaks of 4component NPBs are broader and more flattened (Figures 8(a) and 8(b)).

3.4. Particle Size, Zeta Potential, and Polydispersity Index of NPBs. Dynamic light scattering (DLS) method has been used to measure particle size of materials with dispersed medium of distilled water. The particle size distribution diagram of NPB-R5Ly samples is shown in Figure 9, and the average particle size of the NPB is displayed in Table 5. Most of the NPB samples have more than 2 particle size ranges; the average particle size of those samples are taken to the peak with highest intensity value.

From the collected results, the R5L5 has a smaller and more uniform particle size than others; from 190.1 to $396.1 \mathrm{~nm}$ with the average particle size is $270.5 \pm 18.735 \mathrm{~nm}$ with an intensity of $100.0 \%$. This result can be explained by the significant role of ginsenoside $\mathrm{Rb} 1$ which acts as a particle 


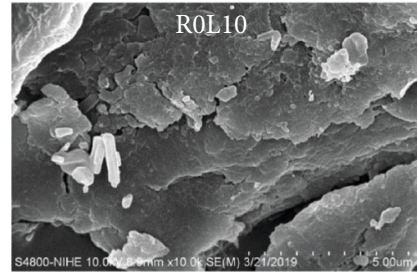

(a)

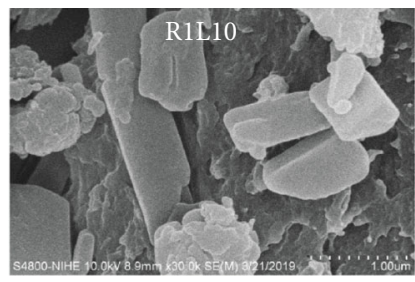

(d)

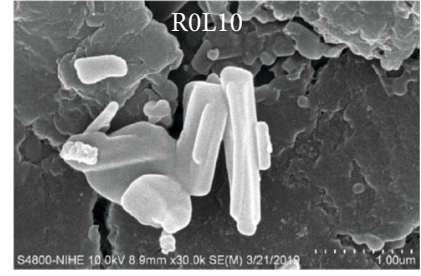

(b)

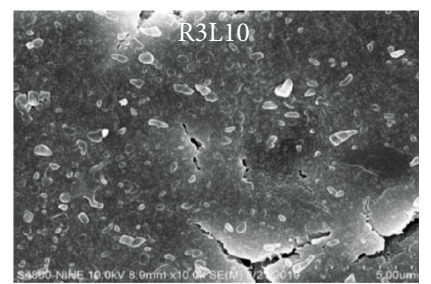

(e)

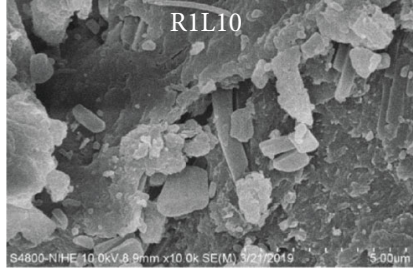

(c)

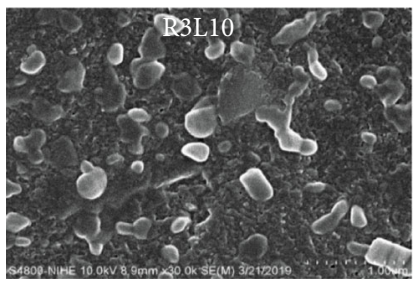

(f)

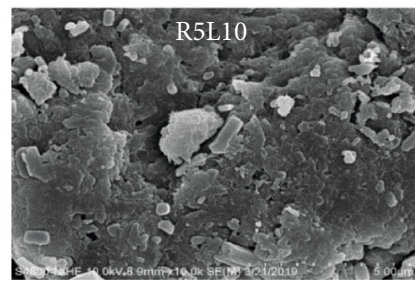

(g)

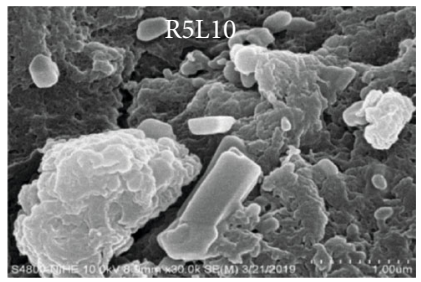

(h)

Figure 6: SEM image of AG/CS/Lov particles (without ginsenoside Rb1) -R0L10 sample and NPBs using 1\%, 3\%, and 5 wt.\% ginsenoside Rb1-R1L10, R3L10, and R5L10 with magnification: $\times 10,000$ (left) and $\times 30,000$ (right).

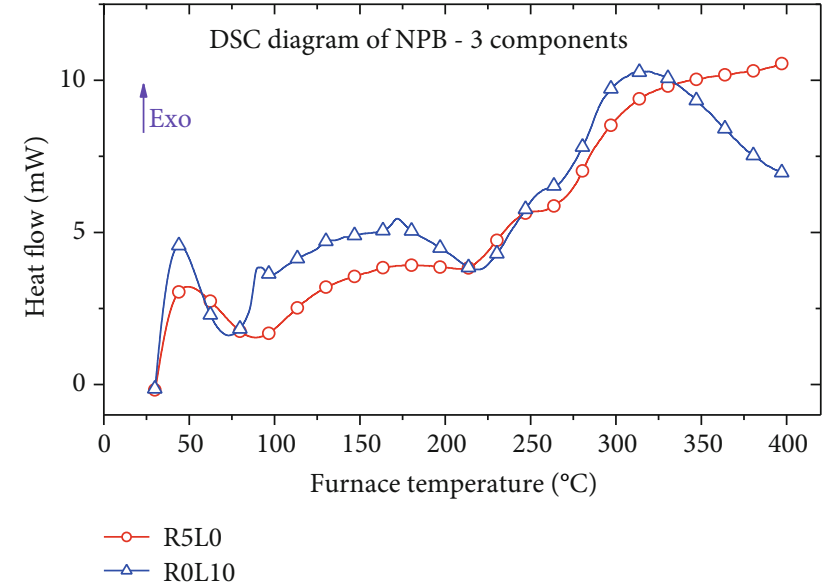

FIgURE 7: DSC diagrams of NPB of R5L0 and R0L10 samples.

size stabilizer in the NPB group. With the appropriate Lov content and the presence of Rb1, based on the interaction between ginsenoside Rb1 with AG, CS, and Lov, the Lov rods disperse more evenly into the AG/CS polymer blend.

Figure 10 and Table 5 show the result of particle size distribution diagram of the NPB group with fixed Lov content and altered ginsenoside $\mathrm{Rb} 1$ content. The average particle size of samples is reduced from $586.8 \pm 61.85 \mathrm{~nm}$ of R0L10, $369.1 \pm 38.46 \mathrm{~nm}$ of R1L10, 328.5 $\pm 68.45 \mathrm{~nm}$ of R3L10, and $333.5 \pm 79.25 \mathrm{~nm}$ of R5L10. So, while increasing ginsenoside $\mathrm{Rb} 1$ content, the average particle size of NPB reduces.
In terms of NPB's particle size, in comparison between FESEM and DLS methods, these values differ between the two methods because in DLS, the particles are dispersed in distilled water; thus, obtained results are much smaller than the FESEM method.

The polydispersity index (PI) which indicates the uniformity of nanoparticles is used to evaluate the heterogeneity of a sample based on size. In accordance with ISO standards (ISO 22,412:2017), PI values $<0.5$ are more common to monodisperse samples, while values $>0.7$ are common to a broad size (e.g., polydisperse) distribution of particles [48]. With the low PDI value mainly ranges from 0.276 to 0.470 , most of the NPBs were found to be stable and monodisperse in nature. The greater PDI values of 0.530-0.595 found in some NPB samples which have broader particle size.

In nano-drug delivery systems, zeta potential/surface charge (ZP) of polymeric particles is an important parameter; it causes major impacts on the various properties of drug delivery polymer particles, for both the stability of the nanoparticles and the rate of drug release. The high absolute value of ZP implies larger repulsive forces between particles, causing difficulty in agglomeration and thus facilitates redispersion of the particles [49]. The ZP values of R0L10, R5L0, R5L10, and R5L20 are -51.3, -23.9, -26.4, and $9.41 \mathrm{mV}$, respectively. It could be seen that the $\mathrm{ZP}$ value of R5L20 is lowest at $9.41 \mathrm{mV}$. The low $\mathrm{ZP}$ value indicates the low resultant repulsion to each other and fast aggregation that consists with the highest particle size of R5L20 sample. In case of the more agglomeration, the higher molecular weight, which 


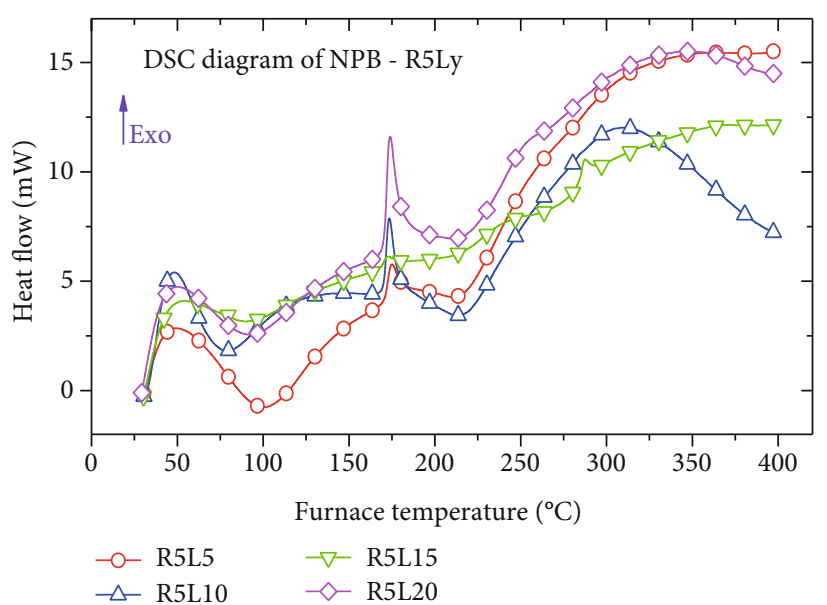

(a)

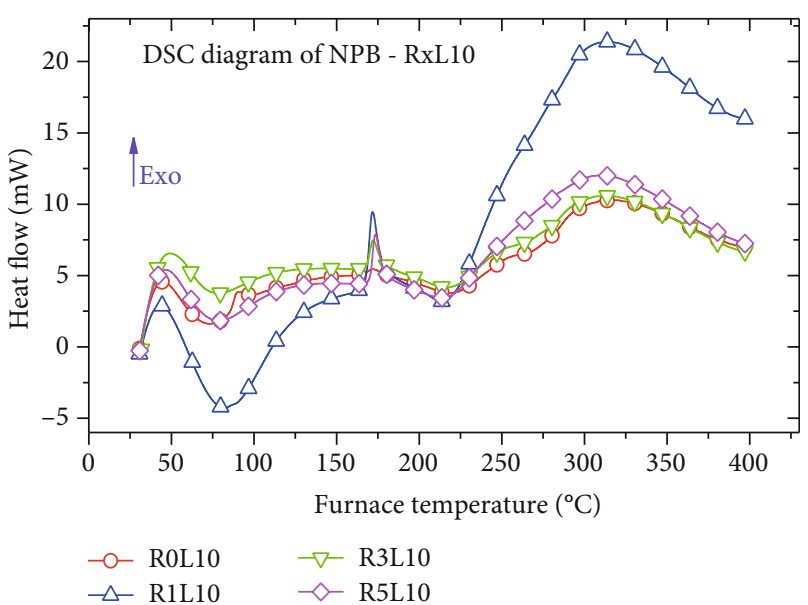

(b)

FIgURE 8: DSC diagrams of NPB with altered Lov content: R5Ly (a) and with altered ginsenoside Rb1 content RxL10 (b).

TABLE 4: Endothermic peak and exothermic peak temperatures of AG, CS, Lov, ginsenoside Rb1, and NPB using different content of Lov and ginsenoside Rb1.

\begin{tabular}{lccc}
\hline Samples & $\begin{array}{c}\text { 1st endothermic } \\
\text { peak temperature } \\
\left({ }^{\circ} \mathrm{C}\right)\end{array}$ & $\begin{array}{c}\text { 1st exothermic } \\
\text { peak } \\
\text { temperature }\left({ }^{\circ} \mathrm{C}\right)\end{array}$ & $\begin{array}{c}\text { 2nd endothermic } \\
\text { peak temperature } \\
\left({ }^{\circ} \mathrm{C}\right)\end{array}$ \\
\hline AG & 119.70 & 238.90 & - \\
CS & 106.80 & - & - \\
Lov & 174.60 & 264.70 & - \\
Rb1 & 98.90 & 230.40 & - \\
R5L0 & 89.51 & - & 214.63 \\
R5L5 & 101.14 & 174.81 & 218.65 \\
R5L10 & 77.35 & 173.47 & 219.31 \\
R5L15 & 91.38 & 172.54 & 219.31 \\
R5L20 & 93.04 & 173.89 & 217.58 \\
R0L10 & 73.24 & 172.14 & 221.74 \\
R1L10 & 82.11 & 171.72 & 219.75 \\
R3L10 & 76.51 & 172.05 & 219.67 \\
\hline
\end{tabular}

steric stabilisation will prevail, a reduction of the measured zeta potential appeared. Hence, despite the low ZP value, the stability of R5L20 is sufficient.

While absolute $\mathrm{ZP}$ values above $30 \mathrm{mV}$ provide good stability and above $60 \mathrm{mV}$ excellent stability for nanoparticles, the stability of R0L10 is excellent, and of R5L0 and R5L10 are considered as good. This might be the result of the firm rod-shape structure of Lov while appearing on the surface of the NPB, leading to the bigger size but stable of the nanoparticle; the higher Lov content, the higher the particle size.

With the presence of Rb1 in NPBs, the particle size is reduced due to the involvement of amorphous structure of Rb1 together with Lov, which could be seen while taking into account the R0L10 and R5L10 samples. ZP values of the two samples indicate the R0L10 is the most stable nanoparticle system.

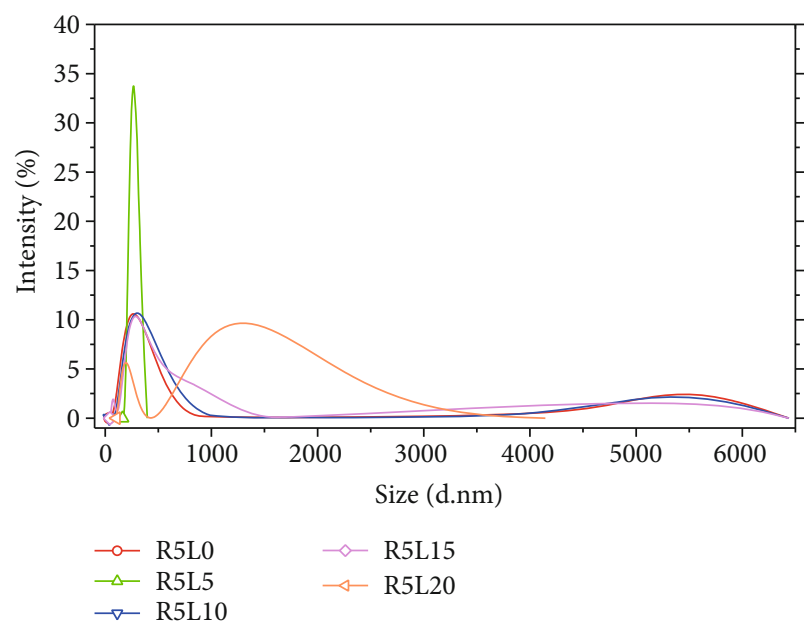

Figure 9: Particle size distribution diagram of NPB using 5 wt.\% ginsenoside Rb1 and different Lov content.

3.5. Loading Efficiency of Lov and Ginsenoside Rb1 by AG/CS Polymer Blend. The loading efficiency of Lov and ginsenoside $\mathrm{Rb} 1$ by AG/CS polymer blend with different initial content of Lov and ginsenoside Rb1 is shown in Table 6. It could be seen that the Lov loading efficiency of the R5Ly group is a bit lower than in the RxL10 group. The highest Lov loading efficiency appeared in the R5L20 sample (78.59\%). For ginsenoside Rb1 loading efficiency, the RxL10 group has a higher result of $71.22 \%, 76.8 \%$, and $73.31 \%$, respectively. From both results, the initial content of ginsenoside Rb1 in NPB has a major impact on Lov and ginsenoside Rb1 loading efficiency.

\subsection{Release of Lov and Ginsenoside Rb1 from NPB Samples in Different $p H$ Solutions}

3.6.1. Influence of $p H$ of Solution on Lov and Ginsenoside Rb1 Released Content from R5L10 Samples. Based on the calculated results of drug release percentage, Lov release process from the R5L10 in both $\mathrm{pH} 2$ and $\mathrm{pH} 7.4$ aqueous solutions 
TABle 5: Particle size of NPB using different Lov and ginsenoside Rb1 content.

\begin{tabular}{|c|c|c|c|c|c|c|}
\hline \multirow[t]{2}{*}{ Sample } & \multirow[t]{2}{*}{ Particle size range $(\mathrm{nm})$} & \multicolumn{2}{|c|}{$\begin{array}{c}\text { Major particle } \\
\text { size }\end{array}$} & \multirow[t]{2}{*}{ Peak width $(r)(\mathrm{nm})$} & \multirow[t]{2}{*}{ Polydispersity index (PDI) } & \multirow{2}{*}{ Average particle size $(\mathrm{nm}) D=d \pm r / 2$} \\
\hline & & $\operatorname{Nm}(d)$ & $\%$ & & & \\
\hline \multirow{2}{*}{ R5L0 } & $43.82-825$ & 285 & 95.1 & 138.2 & 0.470 & $285 \pm 69.1$ \\
\hline & $4145-6400$ & 5272 & 4.9 & 426.7 & & $5272 \pm 213.35$ \\
\hline R5L5 & 190.1-396.1 & 270.5 & 100.0 & 37.47 & 0.460 & $270.5 \pm 18.735$ \\
\hline \multirow{3}{*}{ R5L10 } & $60-90.1$ & 58.43 & 1.5 & 10.36 & 0.346 & $58.4 \pm 5.18$ \\
\hline & $95-1050$ & 333.5 & 93.6 & 159.5 & & $332.5 \pm 79.25$ \\
\hline & $3850-5560$ & 5118.0 & 4.9 & 537 & & $5118.0 \pm 268.50$ \\
\hline \multirow{3}{*}{ R5L15 } & $43.82-190.1$ & 76.86 & 7.2 & 14.52 & 0.595 & $76.86 \pm 7.26$ \\
\hline & $190.1-1718$ & 405.0 & 90.5 & 238.5 & & $405 \pm 119.25$ \\
\hline & $4801-6439$ & 5560.0 & 2.3 & 0.00006 & & $5560 \pm 0.00003$ \\
\hline \multirow{2}{*}{ R5L20 } & $122.4-396.1$ & 204.5 & 28.0 & 52.36 & 0.554 & $204.5 \pm 26.18$ \\
\hline & $458.7-3580$ & 1405.0 & 72.0 & 559.2 & & $1405 \pm 279.6$ \\
\hline R0L10 & $480-1053$ & 586.8 & 100 & 123.7 & 0.483 & $586.8 \pm 61.85$ \\
\hline \multirow{2}{*}{ R1L10 } & $76-150$ & 78.2 & 5.7 & 9.783 & 0.530 & $78.2 \pm 4.89$ \\
\hline & $250-800$ & 369.1 & 94.3 & 76.92 & & $369.1 \pm 38.46$ \\
\hline \multirow{2}{*}{ R3L10 } & $95-950$ & 328.5 & 97.5 & 136.9 & 0.276 & $328.5 \pm 68.45$ \\
\hline & $4000-6500$ & 5274 & 2.5 & 424.6 & & $5274 \pm 212.3$ \\
\hline \multirow{3}{*}{ R5L10 } & $55-90$ & 58.4 & 1.5 & 10.36 & 0.346 & $58.4 \pm 5.18$ \\
\hline & $90-1050$ & 333.5 & 93.6 & 158.5 & & $333.5 \pm 79.25$ \\
\hline & $4500-8500$ & 5118 & 4.9 & 537 & & $5118.0 \pm 268.50$ \\
\hline
\end{tabular}

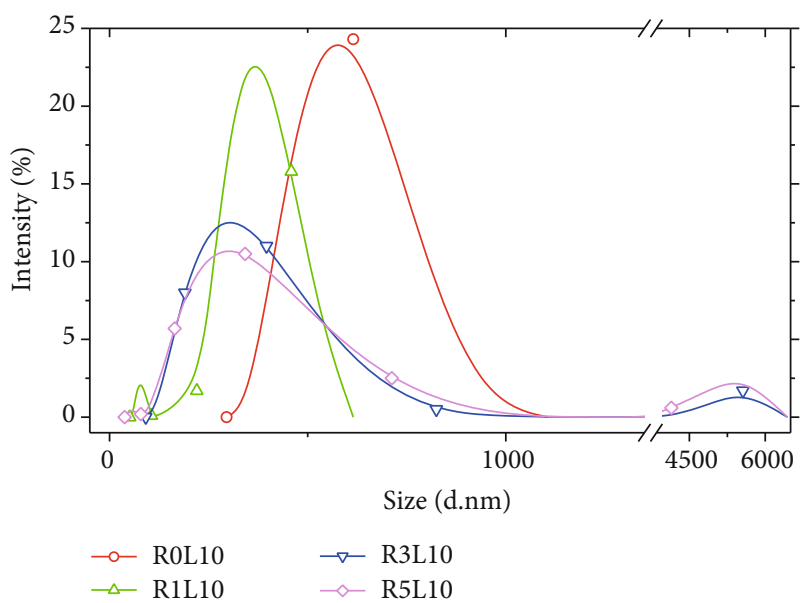

Figure 10: Particle size distribution diagram of NPB using $10 \mathrm{wt} . \%$ Lov and different ginsenoside Rb1 content.

over experiment time has taken place in 2 stages; the release of Lov content has increased significantly during the first 10 hours (about $80 \mathrm{wt} . \%$ ) and reached a stable value in later stage. It is reasonable because at the first stage, at the surface of NPB or closely to the surface, Lov is loaded or attached at high content; thus, it is allowed to quickly be released and strongly dispersed into testing medium. In the next stage,
TABLE 6: Loading efficiency of Lov and ginsenoside Rb1 by AG/CS polymer blend with different initial content of Lov and ginsenoside Rb1.

\begin{tabular}{lcc}
\hline Sample & \multicolumn{2}{c}{$\begin{array}{c}\text { Drug loading efficiency (\%) } \\
\text { Ginsenoside Rb1 }\end{array}$} \\
\hline R5L0 & - & 65.47 \\
R5L5 & 39.25 & 64.21 \\
R5L10 & 70.64 & 73.31 \\
R5L15 & 57.63 & 61.35 \\
R5L20 & 78.59 & 61.87 \\
R0L10 & 62.81 & \\
R1L10 & 61.38 & 71.22 \\
R3L10 & 77.69 & 76.8 \\
\hline
\end{tabular}

Lov rods/particles are trapped deep inside the structure of the NPB and would be harder to access and diffuse outside in different $\mathrm{pH}$ solutions. Moreover, the external solutions are also facing more difficulty to penetrate the grain structure of the NPB due to the swelling, hydrolysis of AG and CS. The $\mathrm{pH}$ of the testing medium influences significantly on the Lov content released from the R5L10 sample (Figure 11). In more detail, after 32 hours, the Lov content released from the NPB in $\mathrm{pH} 2$ solution is $96.99 \%$, while in $\mathrm{pH} 7.4$ solution, it reaches $99.15 \%$. The released Lov content from NPB in 

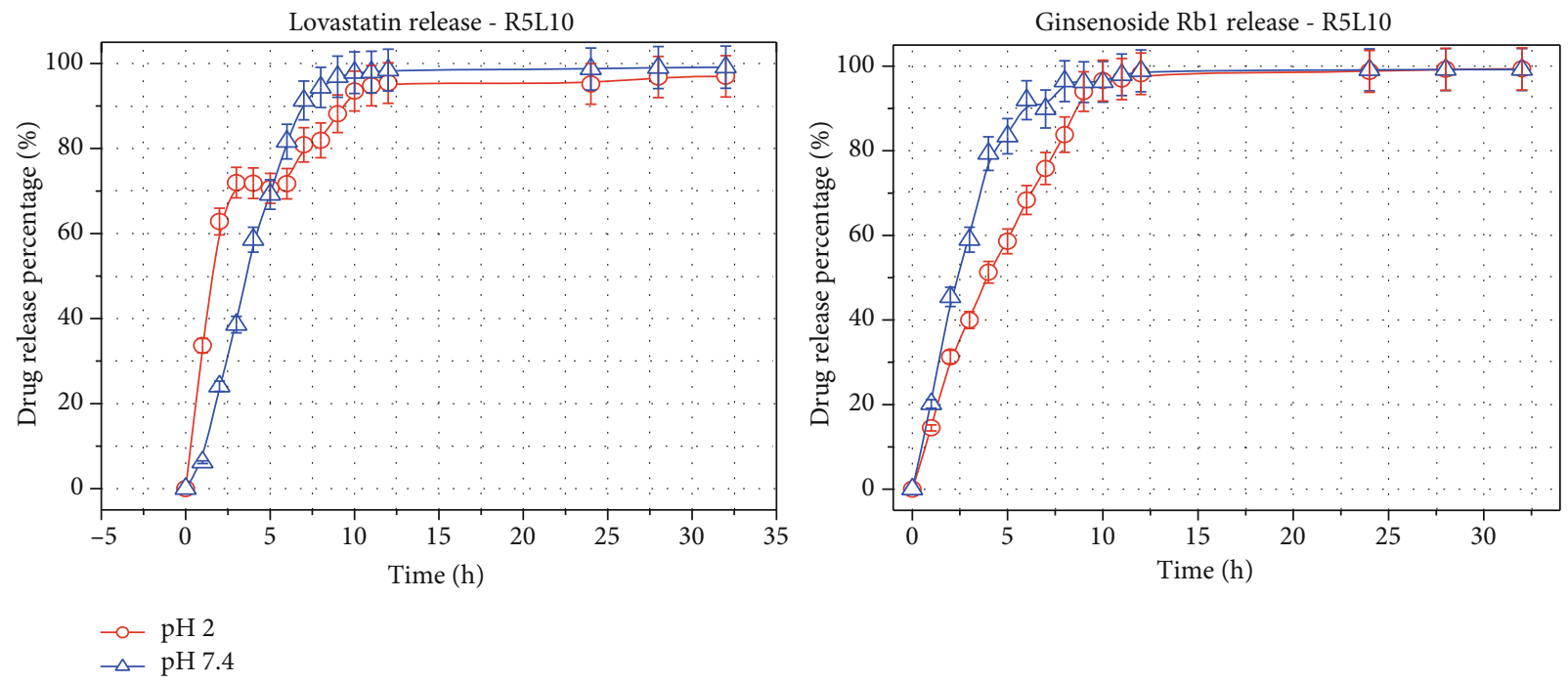

FIgURe 11: Percentage of Lov and ginsenoside Rb1 released from NPB-R5L10 sample in pH 2 and pH 7.4 solutions.
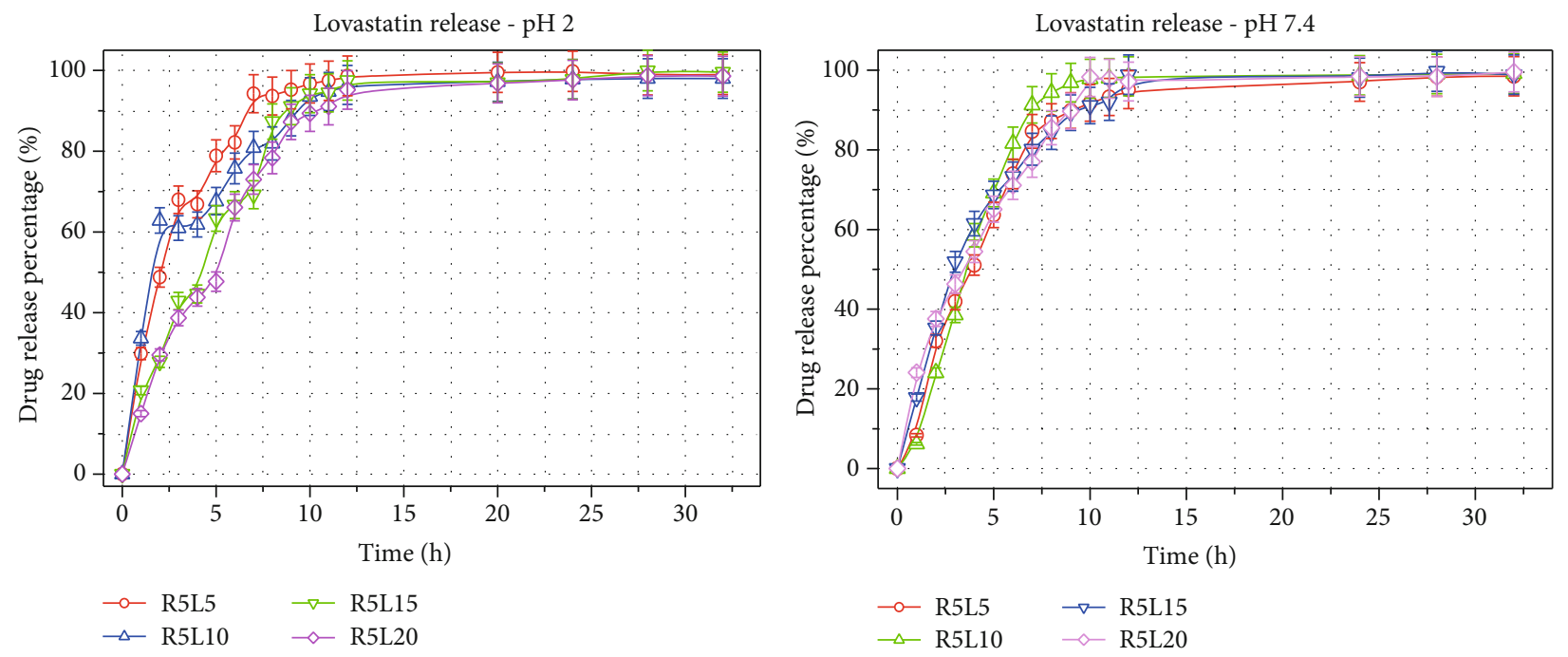

FIGURE 12: Percentage of Lov released from NPB-R5Ly samples in $\mathrm{pH} 2$ and $\mathrm{pH} 7.4$ solutions.

pH 2 solution is lower than in $\mathrm{pH} 7.4$ solution because a portion of hydroxyl group in the released Lov reacts with $\mathrm{H}^{+}$ions in the acid, thus, reducing solubility of Lov in the acid solution. This is consistent with the previous findings in biomedical that Lov is poorly absorbed in the stomach, where the $\mathrm{pH}$ is low. Besides that, the $\mathrm{H}^{+}$ions also react with the amino groups of CS on the surface of the NPB, preventing Lov to diffuse into the solution $[33,34]$. These results demonstrate that the drug-loading polymer particles are more suitable for the neutral environment of the large intestine and rectum mucosa than in acidic environment.

The similar tendency is observed in Figure 11 for the ginsenoside $\mathrm{Rb} 1$ released content from NPB. In $\mathrm{pH} 2$ solution, the released content of $\mathrm{Rb} 1$ increases and reaches $93.60 \%$ for the first 10 hours and remains stable up to $95.36 \%$ after 32 hours. In pH 7.4 solution, this value more quickly reaches $96.30 \%$ within 8 hours and $99.19 \%$ after 32 hours, respec- tively. In neutral environment, the released rate of Rb1 is higher and more stable than in acid environment because the $\mathrm{H}^{+}$ions react with the amino group of CS on the surface, reducing diffusion ability of ginsenoside $\mathrm{Rb} 1$ into the acid. Thus, it is possible to conclude that the $\mathrm{pH}$ of the environment significantly affects the released rate of $\mathrm{Rb} 1$.

3.6.2. Influence of Initial Lov Content on Lov Content Released from NPB Samples. Figure 12 presents the percentage of Lov released from the NPB-R5Ly samples over time in $\mathrm{pH} 2$ and $\mathrm{pH} 7.4$ solutions. Better Lov content released results received with lower initial Lov content among the R5Ly group for the whole experiments in $\mathrm{pH} 2$ with the highest Lov released rate appeared in R5L5 and R5L10 (Figure 12). Might the small particle size help to increase the contact of NPB particles with the solution; therefore, the drug is more easily to dissolve into solutions. Moreover, 

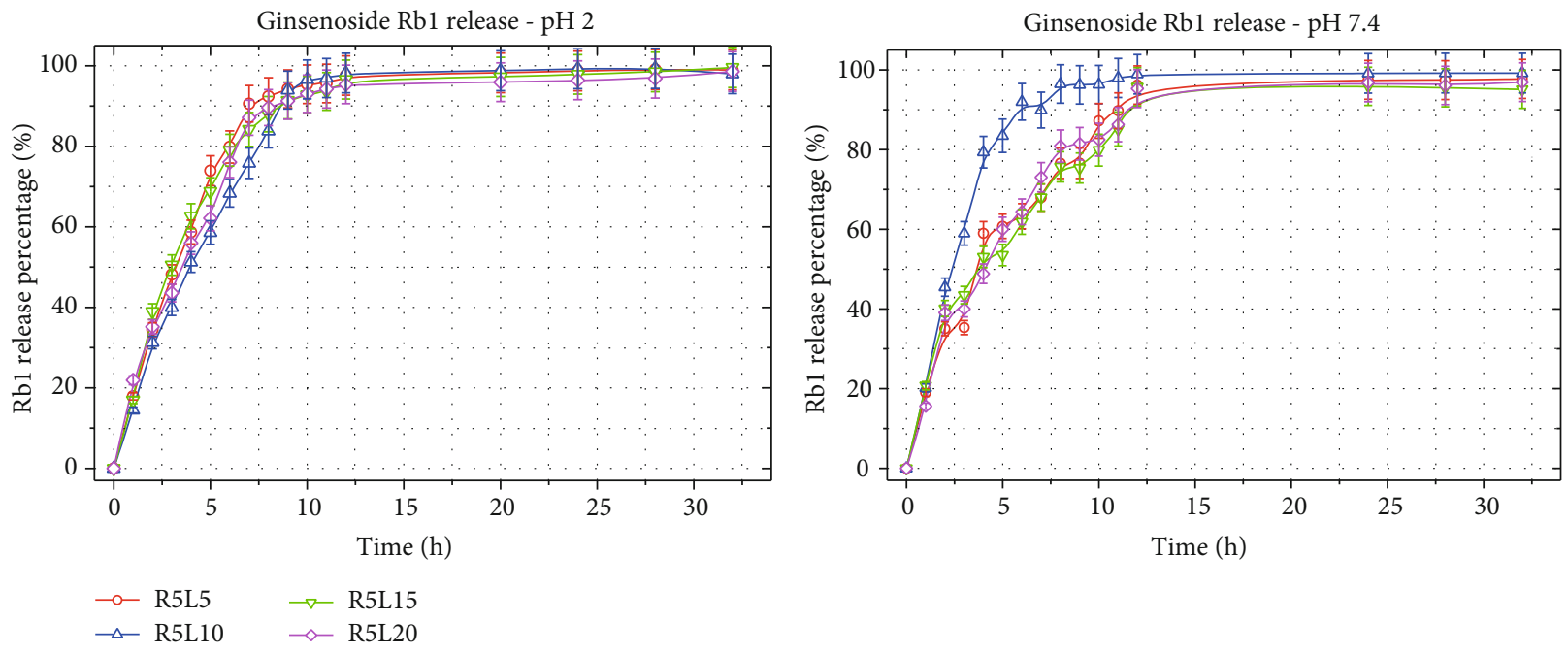

FIGURE 13: Percentage of ginsenoside Rb1 released from NPB-R5Ly samples in $\mathrm{pH} 2$ and $\mathrm{pH} 7.4$ solutions.

at appropriate initial content, the strong interaction between $\mathrm{Rb} 1$ and Lov also causes a synergistic impact in drug release rate.

In pH7.4 solution, the released rate of Lov content is quite similar in the R5Ly group. The difference appeared mainly from 7 to 12 hours, of which, the R5L10 and R5L20 have a bit higher rate than R5L5 and R5L15. This result may be explained by burst release of drugs located on the surface and near the surface of particles at the high content of the drug in neutral environment.

3.6.3. Influence of Initial Lov Content on Ginsenoside Rb1 Content Released from NPB Samples. The dependence of initial Lov content on the percentage of ginsenoside $\mathrm{Rb} 1$ released from the R5Ly group over time in $\mathrm{pH} 2$ and $\mathrm{pH} 7.4$ solutions is shown in Figure 13.

At both pH solutions, contrary to the trend of Lov release rate, the ginsenoside $\mathrm{Rb} 1$ released rate is quite similar at $\mathrm{pH} 2$ and much different at $\mathrm{pH} 7.4$ in all of the R5Ly group. At $\mathrm{pH} 2$, the R5L10 sample has the highest release rate for the first 10 testing hours compared with others; for the rest timing, it has kept a slight change from 1 to $2 \%$, also similar to others. Thus, the ginsenoside $\mathrm{Rb} 1$ content released from the NPB in pH 2 solution is not significantly affected by initial Lov content. In pH 7.4 solution, the ginsenoside Rb1 released rate takes longer time to reach its maximum value (12 hours of experiment) in most of the R5Ly samples; thus, it was still highly impacted by the initial Lov content. The highest result is obtained in the R5L10 sample. This can be explained by the strong interaction of ginsenoside $\mathrm{Rb} 1$ and Lov with polymers which can impact the dispersed phase in polymer matrix and particle size of obtained NPB samples.

This result again reaffirms that ginsenoside Rb1 plays the role as a structural stabilizer, enhancing dispersion and mixing between AG, CS, Lov, and ginsenoside Rb1 as well as itself release ability from the NPB in $\mathrm{pH} 7.4$ solution.

3.6.4. Influence of Initial Ginsenoside Rb1 Content on Lov Content Released from NPB Samples. Figure 14 describes the Lov content released rate from the RxL10 group over time in $\mathrm{pH} 2$ and $\mathrm{pH} 7.4$ solutions. In both cases, during the 8 first hours, Lov content released from the NPBRxL10 samples is increased sharply (over 90\%), then remains in both of the above solutions. According to the shape of the curves and the values of Lov content released rate, similarity has been found for both of $\mathrm{pH}$. In both of $\mathrm{pH}$, the variation of Lov content released from the NPB is quite the same over time. Hence, the change of initial ginsenoside $\mathrm{Rb} 1$ content has a small impact on the Lov released rate in the RxL10 group.

3.6.5. Statistical Evaluation within NPB Groups. Statistical calculation result with Student's $t$-test for data on Figure 12 shows that in the early stage of drug released process, within first 3 hours, the difference of released Lov content between R5Ly samples in $\mathrm{pH} 2$ is highly significant different with those in pH 7.4 in comparing with the chosen level of significance $p=0.05$, with $p$ value ranges from 0.001 to 0.006 , reaching different level at $99-99.8 \%$. In the second stage of Lov released process, from 4 to 8 hours, the different level is decreased with higher $p$ value ranges from 0.054 to 0.168 , with the confidence level of $70-90 \%$. For the rest period, with $p=0.24-0.49$, Lov release percentage is quite similar within the R5Ly samples.

For the released Rb1 content (Figure 13), the R5Ly group has shown its very highly significant different level in $t$-student comparison from the period of 6-11 hours of experiments. During that period, the calculated $p$ value reached 0.004-0.025, with the reliability of evaluation being in the range of $99.8-99.9 \%$. From the first 1-5 hours, the calculated $p$ value is in the range of $0.111-0.293$, with the confidence level of $90-98 \%$; the different level is highly significant. The rest timing of experiments is from 12 to 32 hours with $p=$ $0.075-0.324$, with lower but still being considered as good enough to support a conclusion of $\mathrm{Rb} 1$ released content between the two pHs is at significantly different levels, reaching $90-95 \%$. 

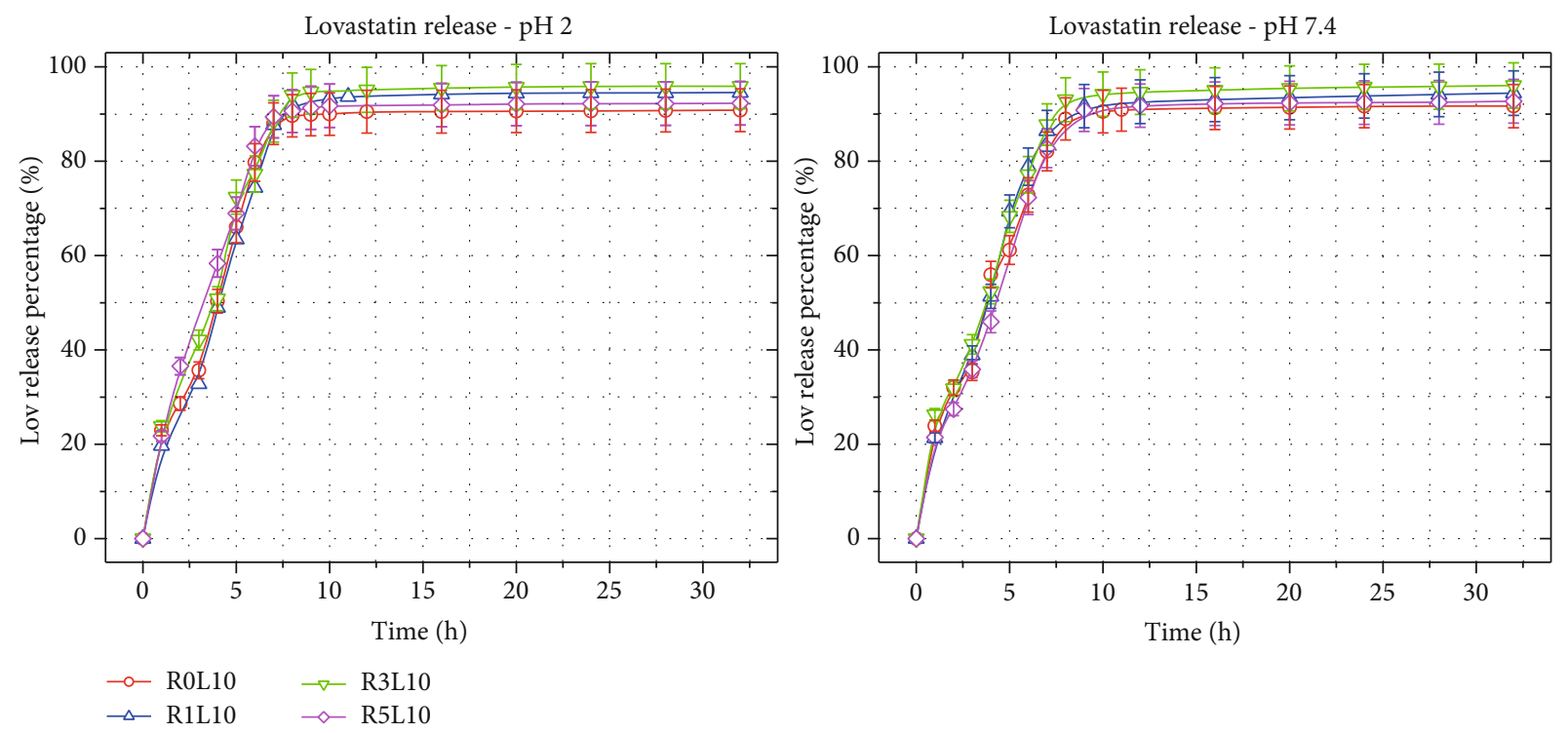

FIgURE 14: Percentage of Lov released from NPB-RxL10 samples in pH 2 and pH 7.4 solutions.

Hence, Lov released content and Rb1 released content of the R5Ly groups are not similar over the same period of experiment timing in each $\mathrm{pH}$ of experiments.

In overall, for every NPB sample, when adding an amount of ginsenoside Rb1 to the AG/CS/Lov particles, the Lov content released from the NPB sample is increased in both $\mathrm{pH} 2$ and $\mathrm{pH} 7.4$ solutions. In acidic environment, the released Lov content is lower than in neutral environment. Following the results, it could be suggested that 4component NPB is suitable for use in the environment of intestinal and gastric fluid in human.

\section{Conclusions}

The novel nanoparticle biomaterials (NPB) of alginate/chitosan (AG/CS) polymer blend loading simultaneously lovastatin (Lov) and ginsenoside Rb1 were successfully fabricated by ionic gelation method using $\mathrm{CaCl}_{2}$ and sodium tripolyphosphate as gelation agent and cross-linking agent, respectively. The results of FTIR analysis of NPB samples using different Lov and ginsenoside Rb1 content show that the peak position of the characterized groups in AG, CS, Lov, and ginsenoside Rb1 has shifted slightly due to the mutual interaction such as dipole-dipole interactions and hydrogen bonding between the characteristic groups in AG, $\mathrm{CS}$, Lov, and ginsenoside Rb1. As a particle size stabilizer for AG/CS/Lov particles, ginsenoside Rb1 contributes to dispersing Lov rods more regularly into the AG/CS polymer blend. Among the investigated NPB samples, the NPB with AG/CS ratio of 1/1 (wt./wt.), 5 wt.\% Lov and 5 wt.\% ginsenoside Rb1 (R5L5) sample has a particle size in the range from 190.1 to $396.1 \mathrm{~nm}$; the average particle size is $270.5 \pm$ $18.735 \mathrm{~nm}$ with an intensity of $100.0 \%$. Zeta potential values indicate the excellent stability of R0L10 and good stability of R5L0 and R5L10 which means the 4-components with 5 wt.\% Rb1 and 10 wt.\% Lov content is the decent stable nanoparticle system. The Lov release process from the NPB samples in both $\mathrm{pH} 2$ and $\mathrm{pH} 7.4$ solutions according to testing time includes 2 stages, quick release for the first testing 10 hours, then slow release to stable value. Similarly, the ginsenoside $\mathrm{Rb} 1$ release process from the NPB samples in both $\mathrm{pH} 2$ and pH 7.4 solutions also includes 2 stages, quick release for the first testing 7 hours, then slow down to a stable value. The release of Lov and ginsenoside Rbl content from the $\mathrm{NPB}$ samples in $\mathrm{pH} 2$ solution is lower than in $\mathrm{pH} 7.4$ solution with the same components of NPB samples and testing time.

\section{Data Availability}

Data availability statement is included in the paper content.

\section{Conflicts of Interest}

There are no conflicts to declare.

\section{Acknowledgments}

The authors would like to thank the National Foundation for Science and Technology Development in Vietnam for financial support (subject code 104.02-2017.17, period of 20172020).

\section{References}

[1] W. Paul and C. P. Sharma, "Alginates: wound dressings," in Encyclopedia of Biomedical Polymers and Polymeric Biomaterials, 2015.

[2] E. C. Stanford, "Improvements in the manufacture of useful products from seaweeds," Tech. Rep. 142, British patent, 1881.

[3] K. Y. Lee and D. J. Mooney, "Alginate: properties and biomedical applications," Progress in Polymer Science, vol. 37, no. 1, pp. 106-126, 2012.

[4] W. Sabra and W.-D. Deckwer, Alginate-A Polysaccharide of Industrial Interest and Diverse Biological Functions, in 
Polysaccharides-Structural Diversity and Functional Versatility, Marcel Dekker, New York, 2nd edition, 2005.

[5] Y. Pranoto, V. M. Salokhe, and S. K. Rakshit, "Physical and antibacte rial properties of alginate-based edible film incorporated with garlic oil," Food Research International, vol. 38, no. 3, pp. 267-272, 2005.

[6] K. Norajit and G. H. Ryu, "Preparation and properties of antibacterial alginate films incorporating extruded white ginseng extract," Journal of Food Processing and Preservation, vol. 35, no. 4, pp. 387-393, 2011.

[7] A. Mohammed Fayaz, "Mycobased synthesis of silver nanoparticles and their incorporation into sodium alginate films for vegetable and fruit preservation," Journal of Agricultural and Food Chemistry, vol. 57, no. 14, pp. 6246-6252, 2009.

[8] A. Concha-Meyer, R. Schöbitz, C. Brito, and R. Fuentes, "Lactic acid bacteria in an alginate film inhibit Listeria monocytogenes_ growth on smoked salmon," Food Control, vol. 22, no. 3-4, pp. 485-489, 2011.

[9] M. Joyce, R. Gilbert, and S. Khan, "Effect of Ca++ ions on the water retention of alginate in paper coatings," Journal of Pulp and Paper Science, vol. 22, no. 4, pp. J126-J130, 1996.

[10] A. Hambleton, F. Debeaufort, A. Bonnotte, and A. Voilley, "Influence of alginate emulsion-based films structure on its barrier properties and on the protection of microencapsulated aroma compound," Food Hydrocolloids, vol. 23, no. 8, pp. 2116-2124, 2009.

[11] L. P. da Costa, "Alginate in biomedical applications," in Alginates, p. 81, 2019.

[12] Y. Qin, "The gel swelling properties of alginate fibers and their applications in wound management," Polymers for Advanced Technologies, vol. 19, no. 1, pp. 6-14, 2008.

[13] T. Gilchrist and A. Martin, "Wound treatment with Sorbsan an alginate fibre dressing," Biomaterials, vol. 4, no. 4, pp. 317$320,1983$.

[14] R. Fraser and T. Gilchrist, "Sorbsan calcium alginate fibre dressings in footcare," Biomaterials, vol. 4, no. 3, pp. 222224, 1983.

[15] C. Knill, J. Kennedy, J. Mistry et al., "Alginate fibres modified with unhydrolysed and hydrolysed chitosans for wound dressings," Carbohydrate Polymers, vol. 55, no. 1, pp. 65-76, 2004.

[16] P. Yadav, H. Yadav, V. G. Shah, G. Shah, and G. Dhaka, "Biomedical biopolymers, their origin and evolution in biomedical sciences: a systematic review," Journal of Clinical and Diagnostic Research: JCDR, vol. 9, no. 9, pp. ZE21-ZE25, 2015.

[17] F. Shahidi, J. K. V. Arachchi, and Y.-J. Jeon, "Food applications of chitin and chitosans," Trends in Food Science \& Technology, vol. 10, no. 2, pp. 37-51, 1999.

[18] S. Hirano, "Chitin biotechnology applications," in Biotechnology annual review, pp. 237-258, Elsevier, 1996.

[19] R. Yadav and M. Chauhan, "Pharmaceutical diversity of chitin and chitosan: a review," International Journal of Pharmaceutical Sciences and Research, vol. 2, pp. 6-11, 2017.

[20] L. Ilium, "Chitosan and its use as a pharmaceutical excipient," Pharmaceutical Research, vol. 15, no. 9, pp. 1326-1331, 1998.

[21] G. W. Gooday, "The ecology of chitin degradation," in Advances in microbial ecology, pp. 387-430, Springer, 1990.

[22] S. E. Bailey, T. J. Olin, R. M. Bricka, and D. D. Adrian, "A review of potentially low-cost sorbents for heavy metals," Water Research, vol. 33, no. 11, pp. 2469-2479, 1999.
[23] A. Singla and M. Chawla, "Chitosan: some pharmaceutical and biological aspects-an update," Journal of Pharmacy and Pharmacology, vol. 53, no. 8, pp. 1047-1067, 2001.

[24] S. Koide, "Chitin-chitosan: properties, benefits and risks," Nutrition Research, vol. 18, no. 6, pp. 1091-1101, 1998.

[25] V. Dodane and V. D. Vilivalam, "Pharmaceutical applications of chitosan," Pharmaceutical Science \& Technology Today, vol. 1, no. 6, pp. 246-253, 1998.

[26] Y. Sun, J. Zhang, S. Wu, and Shujun Wang, "Preparation of Dglucosamine by hydrolysis of chitosan with chitosanase and $\beta$ D-glucosaminidase," International Journal of Biological Macromolecules, vol. 61, pp. 160-163, 2013.

[27] L. Dai Tran, H. V. Tran, T. T. Mai et al., "Biomedical and environmental applications of chitosan-based nanomaterials," Journal of Chitin chitosan, vol. 16, no. 1, p. 7, 2011.

[28] J. Liu, J. Zhang, Y. Shi, S. Grimsgaard, T. Alraek, and V. Fønnebø, "Chinese red yeast rice (Monascus purpureus) for primary hyperlipidemia: a meta-analysis of randomized controlled trials," Chinese Medicine, vol. 1, no. 1, p. 4, 2006.

[29] N. Gunde-Cimerman and A. Cimerman, "Pleurotus fruiting bodies contain the inhibitor of 3-hydroxy-3-methylglutarylcoenzyme A reductase-lovastatin," Experimental Mycology, vol. 19, no. 1, pp. 1-6, 1995.

[30] R. V. Fugit and N. D. Resch, "Conversion of patients from simvastatin to lovastatin in an outpatient pharmacy clinic," American Journal of Health-System Pharmacy, vol. 57, no. 18, pp. 1703-1708, 2000.

[31] A. J. Shinde and H. N. More, "Lovastatin loaded chitosan nanoparticles: preparation, evaluation and in vitro release studies," Research Journal of Pharmacy and Technology, vol. 4, no. 12, pp. 1869-1876, 2011.

[32] C.-Y. Yu, X.-C. Zhang, F. Z. Zhou, X. Z. Zhang, S. X. Cheng, and R. X. Zhuo, "Sustained release of antineoplastic drugs from chitosan-reinforced alginate microparticle drug delivery systems," International Journal of Pharmaceutics, vol. 357, no. 1-2, pp. 15-21, 2008.

[33] N. S. Rejinold, M. Muthunarayanan, K. Muthuchelian, K. P. Chennazhi, S. V. Nair, and R. Jayakumar, "Saponin-loaded chitosan nanoparticles and their cytotoxicity to cancer cell lines in vitro," Carbohydrate Polymers, vol. 84, no. 1, pp. 407-416, 2011.

[34] R. Nanda, A. Sasmal, and P. Nayak, "Preparation and characterization of chitosan-polylactide composites blended with Cloisite 30B for control release of the anticancer drug paclitaxel," Carbohydrate Polymers, vol. 83, no. 2, pp. 988-994, 2011.

[35] P. Li, Y.-N. Dai, J. P. Zhang, A. Q. Wang, and Q. Wei, “Chitosan-alginate nanoparticles as a novel drug delivery system for nifedipine," International journal of biomedical science: IJBS, vol. 4, no. 3, pp. 221-228, 2008.

[36] L. Lacerda, A. L. Parize, V. Fávere, M. C. M. Laranjeira, and H. K. Stulzer, "Development and evaluation of $\mathrm{pH}$-sensitive sodium alginate/chitosan microparticles containing the antituberculosis drug rifampicin," Materials Science and Engineering: $C$, vol. 39, pp. 161-167, 2014.

[37] A. García-Ceja, E. Mani-López, E. Palou, and A. López-Malo, "Viability during refrigerated storage in selected food products and during simulated gastrointestinal conditions of individual and combined lactobacilli encapsulated in alginate or alginatechitosan," LWT - Food Science and Technology, vol. 63, no. 1, pp. 482-489, 2015. 
[38] N. T. Chinh, N. T. Ly, T. T. Mai et al., "Characteristic and properties of chitosan/alginate polymer blend carrying lovastatin drug," Journal of Science and Technology, vol. 54, no. 2B, pp. 118-124, 2016.

[39] H. Thai, C. Thuy Nguyen, L. Thi Thach et al., "Characterization of chitosan/alginate/lovastatin nanoparticles and investigation of their toxic effects _in vitro_ and _in vivo_," Scientific Reports, vol. 10, no. 1, pp. 909-915, 2020.

[40] H. Zhang, Z. Li, Z. Zhou, H. Yang, Z. Zhong, and C. Lou, "Antidepressant-like effects of ginsenosides: a comparison of ginsenoside $\mathrm{Rb}_{3}$ and its four deglycosylated derivatives, $\mathrm{Rg}_{3}$, $\mathrm{Rh}_{2}$, compound $\mathrm{K}$, and 20(_S_)-protopanaxadiol in mice models of despair," Pharmacology Biochemistry and Behavior, vol. 140, pp. 17-26, 2016.

[41] W. Xie, X. Meng, Y. Zhai et al., "Panax notoginseng saponins: a review of its mechanisms of antidepressant or anxiolytic effects and network analysis on phytochemistry and pharmacology," Molecules, vol. 23, no. 4, p. 940, 2018.

[42] H. Xiang, Y. Liu, B. Zhang et al., "The antidepressant effects and mechanism of action of total saponins from the caudexes and leaves of Panax notoginseng in animal models of depression," Phytomedicine, vol. 18, no. 8-9, pp. 731-738, 2011.

[43] P. Su, L. Wang, S. J. du, W. F. Xin, and W. S. Zhang, "Advance in studies of Panax notoginseng saponins on pharmacological mechanism of nervous system disease," China journal of Chinese materia medica, vol. 39, no. 23, pp. 4516-4521, 2014.

[44] R. Bahramsoltani, M. H. Farzaei, M. S. Farahani, and R. Rahimi, "Phytochemical constituents as future antidepressants: a comprehensive review," Reviews in the Neurosciences, vol. 26, no. 6, pp. 699-719, 2015.

[45] H. Thai, D. L. Tran, T. L. Thach et al., "Effect of both lovastatin and ginsenoside $\mathrm{Rb} 1$ on some properties and in-vitro drug release of alginate/chitosan/lovastatin/ginsenoside $\mathrm{Rb} 1$ composite films," Journal of Polymers and the Environment, vol. 27, no. 12, pp. 2728-2738, 2019.

[46] T. Hoang, K. Ramadass, T. T. Loc et al., "Novel drug delivery system based on ginsenoside Rb1 loaded to chitosan/alginate nanocomposite films," Journal of Nanoscience and Nanotechnology, vol. 19, no. 6, pp. 3293-3300, 2019.

[47] D.-T. Nghiem, T.-C. Nguyen, M. T. Do et al., "Influence of the preparation method on some characteristics of alginate/chitosan/lovastatin composites," Advances in Polymer Technology, vol. 2020, Article ID 7879368, 12 pages, 2020.

[48] G. Kandav, D. Bhatt, and D. K. Jindal, "Formulation and evaluation of allopurinol loaded chitosan nanoparticles," International Journal of Applied Pharmaceutics, vol. 11, no. 3, pp. 49-52, 2019.

[49] S. Honary and F. Zahir, "Effect of zeta potential on the properties of nano-drug delivery systems - a review (part 1)," Tropical Journal of Pharmaceutical Research, vol. 12, no. 2, pp. 265273, 2013. 